\title{
A GENERALIZATION OF A NECESSARY AND SUFFICIENT CONDITION FOR PRIMALITY DUE TO VANTIEGHEM
}

\author{
L. J. P. KILFORD
}

Received 10 March 2004

We present a family of congruences which hold if and only if a natural number $n$ is prime.

2000 Mathematics Subject Classification: 11A51, 11A07.

The subject of primality testing has been in the mathematical and general news recently, with the announcement [1] that there exists a polynomial-time algorithm to determine whether an integer $p$ is prime or not.

There are older deterministic primality tests which are less efficient; the classical example is Wilson's theorem, that

$$
(n-1) ! \equiv-1 \bmod n
$$

if and only if $n$ is prime. Although this is a deterministic algorithm, it does not provide a workable primality test because it requires much more calculation than trial division.

This note provides another family of congruences satisfied by primes and only by primes; it is a generalization of previous work. They could be used as examples of primality tests for students studying elementary number theory.

In Guy [3, Problem A17], the following result due to Vantieghem [4] is quoted as follows.

THEOREM 1 (Vantieghem [4]). Let $n$ be a natural number greater than 1 . Then $n$ is prime if and only if

$$
\prod_{d=1}^{n-1}\left(1-2^{d}\right) \equiv n \bmod \left(2^{n}-1\right) .
$$

In this note, we will generalize this result to obtain the following theorem.

THEOREM 2. Let $m$ and $n$ be natural numbers greater than 1 . Then $n$ is prime if and only if

$$
\prod_{d=1}^{n-1}\left(1-m^{d}\right) \equiv n \bmod \frac{m^{n}-1}{m-1} .
$$

We note that these congruences are also much less efficient than trial division.

Proof. We follow the method of Vantieghem, using a congruence satisfied by cyclotomic polynomials. 
LEMMA 3 (Vantieghem). Let $m$ be a natural number greater than 1 and let $\Phi_{m}(X)$ be the mth cyclotomic polynomial. Then

$$
\prod_{\substack{d=1 \\(d, m)=1}}^{m}\left(X-Y^{d}\right) \equiv \Phi_{m}(X) \bmod \Phi_{m}(Y) \quad \text { in } \mathbb{Z}[X, Y] .
$$

Proof of Lemma 3. We can write

$$
\prod_{\substack{d=1 \\(d, m)=1}}^{m}\left(X-Y^{d}\right)-\Phi_{m}(X)=f_{0}(Y)+f_{1}(Y) X+f_{2}(Y) X^{2}+\cdots .
$$

(Here the $f_{i}$ are polynomials over $\mathbb{Z}$.)

Let $\zeta$ be a primitive $m$ th root of unity. Now, if $Y=\zeta$, then we see that the left-hand side of this expression is identically 0 in $X$.

This implies that the $f_{i}$ are zero at every $\zeta$ and every $i$. Therefore, we have $f_{i}(Y) \equiv$ $0 \bmod \Phi_{m}(Y)$, which is enough to prove the lemma.

Suppose that the natural number $n$ in Theorem 2 is prime. Let $p:=n$. We have that $\Phi_{p}(X)=X^{p-1}+X^{p-2}+\cdots+X+1$. Therefore, if we set $m=p$ in Lemma 3 , we find that

$$
\prod_{d=1}^{p-1}\left(X-Y^{d}\right) \equiv X^{p-1}+X^{p-2}+\cdots+X+1 \bmod \left(Y^{p-1}+\cdots+1\right) .
$$

We now set $X=1$ and $Y=m$, to get

$$
\prod_{d=1}^{p-1}\left(1-m^{d}\right) \equiv p \bmod \frac{m^{p}-1}{m-1}
$$

This proves that if $p$ is prime, then the congruence holds.

We now prove the converse, by supposing that the congruence (3) holds, and that $p$ is not prime. Therefore $p$ is composite, and hence has a smallest prime factor $q$. We write $p=q \cdot a$; now $q \leq a$, and also $p \leq a^{2}$.

Now we have that $m^{a}-1$ divides $m^{p}-1$ and $m^{a}-1$ divides the product $\prod_{d=1}^{p-1}\left(m^{d}-\right.$ $1)$. By combining this with the congruence (3) in Theorem 2 , this implies that $\left(m^{a}-\right.$ 1) / $(m-1)$ divides $p$. Therefore we have

$$
2^{a}-1 \leq \frac{m^{a}-1}{m-1} \leq p \leq a^{2} .
$$

The inequality $2^{a}-1 \leq a^{2}$ forces $a$ to be either 2 or 3 ; this means that $p \in\{4,6,9\}$ and $m \in\{2,3\}$; one can check by hand that the congruence does not hold in this case, so we have proved Theorem 2 .

Guy also asks if there is a relationship between the congruence given by Vantieghem and Wilson's theorem. The following theorem gives an elementary congruence similar to that of Vantieghem between a product over integers and a cyclotomic polynomial. It is in fact equivalent to Wilson's theorem. 
THEOREM 4. Let $m$ be a natural number greater than 2. Define the product $F(X)$ by

$$
F(X):=\prod_{\substack{i=1 \\(i, m)=1}}^{m-1}(X-i-1)+1
$$

Then $m$ is prime if and only if

$$
\Phi_{m}(X) \equiv F(X) \bmod m
$$

Proof of THeOrem 4. Firstly, we prove that if $m$ is not prime, the congruence (10) in Theorem 4 does not hold.

Recall that $\phi(m)$ is defined to be Euler's totient function; the number of integers in the set $\{1, \ldots, m\}$ which are coprime to $m$.

The coefficient of $X^{\phi(m)-1}$ in $F(X)$ is given by the sum

$$
-\sum_{\substack{i=1 \\(i, m)=1}}^{m-1}(i+1)=-\phi(m)-\sum_{\substack{i=1 \\(i, m)=1}}^{m-1} i .
$$

We find that the following congruence holds:

$$
-\phi(m)-\sum_{\substack{i=1 \\(i, m)=1}}^{m-1} i \equiv-\phi(m) \bmod m .
$$

This follows from the following identity:

$$
\sum_{\substack{i=1 \\(i, m)=1}}^{m-1} i=\frac{m \phi(m)}{2}
$$

Because $m>2, \phi(m)$ is divisible by 2, the sum on the left-hand side of (12) is a multiple of $m$. We now use some theorems to be found in a paper by Gallot [2, Theorems 1.1 and 1.4].

THEOREM 5. Let $p$ be a prime and $m$ a natural number.

(1) The following relations between cyclotomic polynomials hold:

$$
\Phi_{p m}(x)= \begin{cases}\Phi_{m}\left(x^{p}\right) & \text { if } p \mid m, \\ \frac{\Phi_{m}\left(x^{p}\right)}{\Phi_{m}(x)} & \text { if } p \nmid m .\end{cases}
$$

(2) If $m>1$, then

$$
\Phi_{n}(1)= \begin{cases}p & \text { if } n \text { is a power of a prime } p \\ 1 & \text { otherwise }\end{cases}
$$


From these results, we see that if $m$ is not a prime power, we then have $\Phi_{n}(1) \equiv$ $1 \bmod m$, and $F(1)$ is given by

$$
1+\prod_{\substack{i=1 \\(i, m)=1}}^{m-1}(-i)
$$

We see that this is not congruent to $1 \bmod m$ because the product is over those $i$ which are coprime to $m$, so the product does not vanish modulo $m$.

If $m$ is a prime power $p^{n}$, then we see from Theorem 5 that $\Phi_{p^{n}}(x)=\Phi_{p}\left(x^{p^{n-1}}\right)$; in particular, we see that the coefficient of $x^{\phi\left(p^{n}\right)-1}$ is 0 , which differs from the coefficient of $x^{\phi\left(p^{n}\right)-1}$ in $F(X)$.

Therefore, if $m$ is not prime, then the congruence does not hold. We now show that if $m$ is prime, the congruence holds.

If $m$ is prime, then $\Phi_{m}(x)=x^{m-1}+x^{m-2}+\cdots+x+1$. We consider the polynomials $\Phi_{m}(X+1)$ and $F(X+1)$. Now, modulo $m$ we have

$$
\Phi_{m}(X+1)=X^{m-1}, \quad F(X+1)=\prod_{\substack{i=1 \\(i, m)=1}}^{m-1}(X-i)+1 .
$$

Now if $x \neq 0 \bmod m$, then we see that $\Phi_{m}(x+1) \equiv 1$ and that $F(x+1) \equiv 1$, because the product vanishes.

And if we have $x=0$, then $\Phi_{m}(x)=0$ and, by Wilson's theorem, $F(0) \equiv(m-1) !+1 \equiv$ $0 \bmod m$.

Therefore we have proved Theorem 4 .

\section{REFERENCES}

[1] M. Agrawal, N. Kayal, and N. Saxena, PRIMES is in P, preprint, 2002, http://www.cse.iitk. ac.in/news/primality.html.

[2] Y. Gallot, Cyclotomic polynomials and prime numbers, preprint, 2001, http://perso. wanadoo.fr/yves.gallot/papers.

[3] R. K. Guy, Unsolved Problems in Number Theory, 2nd ed., Problem A17. Problem Books in Mathematics, I, Springer-Verlag, New York, 1994.

[4] E. Vantieghem, On a congruence only holding for primes, Indag. Math. (N.S.) 2 (1991), no. 2, 253-255.

L. J. P. Kilford: The University of Oxford, Mathematical Institute, 24-29 Street Giles', Oxford OX1 3LB, UK 


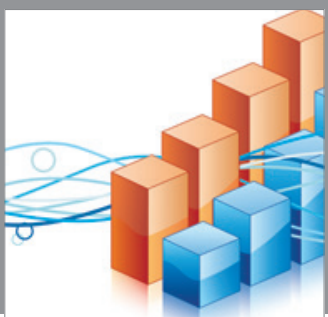

Advances in

Operations Research

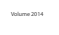

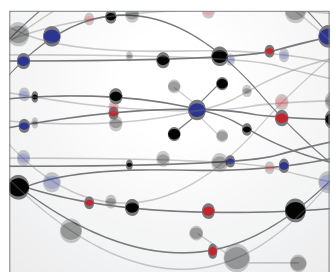

\section{The Scientific} World Journal
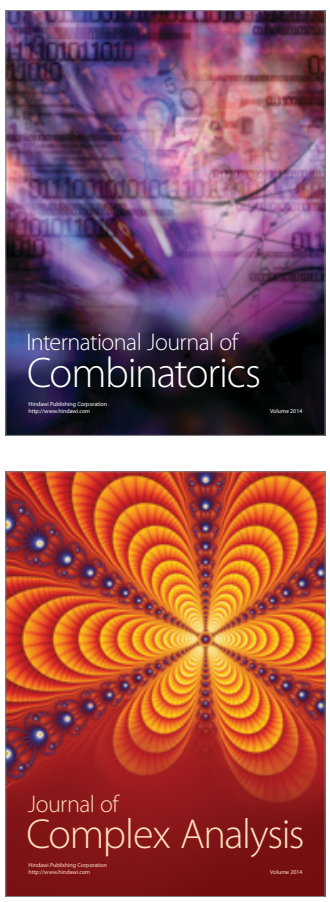

International Journal of

Mathematics and

Mathematical

Sciences
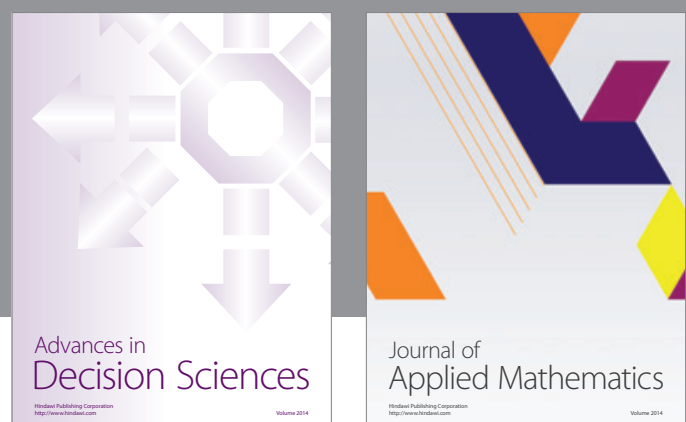

Journal of

Applied Mathematics
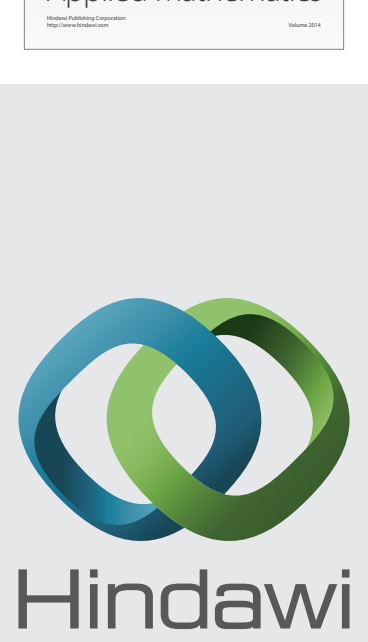

Submit your manuscripts at http://www.hindawi.com
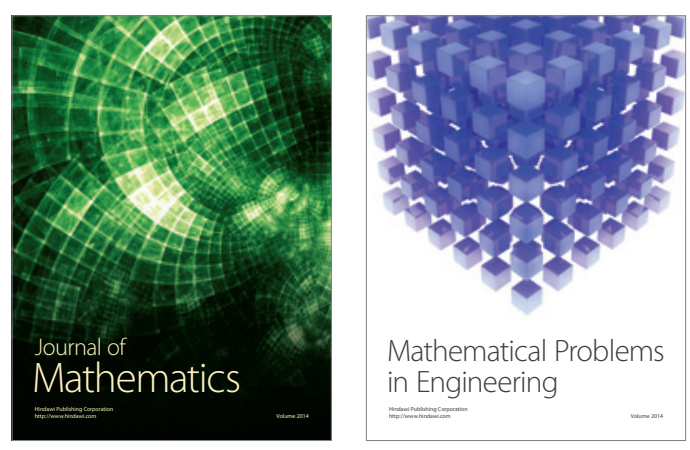

Mathematical Problems in Engineering
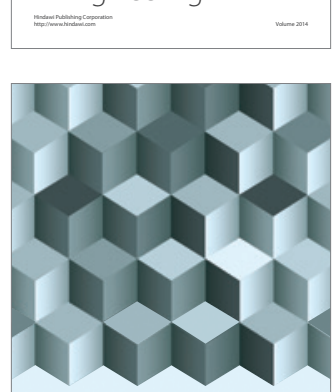

Journal of

Function Spaces
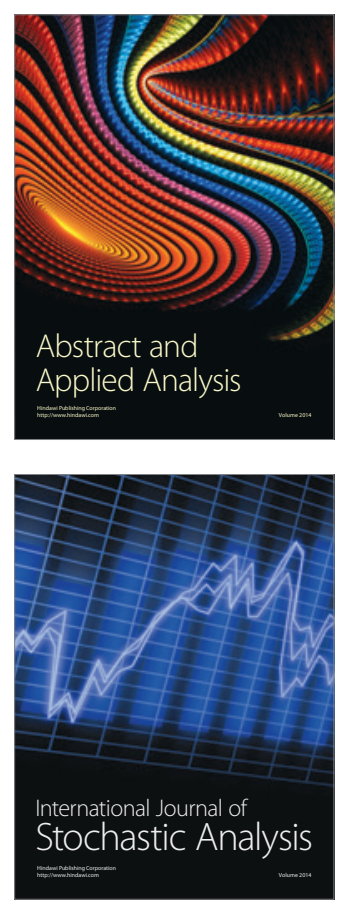

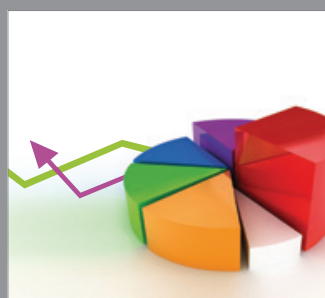

ournal of

Probability and Statistics

Promensencen
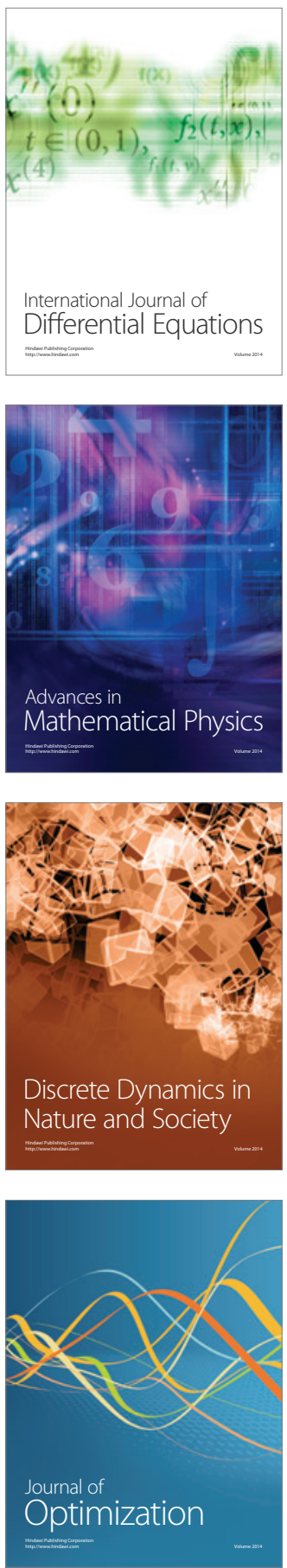